\title{
Review Article \\ Chemokines and Cytokines as Salivary Biomarkers for the Early Diagnosis of Oral Cancer
}

\author{
Gareema Prasad and Michael McCullough \\ Melbourne Dental School, The University of Melbourne, 720 Swanston Street, Carlton, Parkville, VIC 3010, Australia \\ Correspondence should be addressed to Michael McCullough; m.mccullough@unimelb.edu.au
}

Received 16 July 2013; Accepted 31 October 2013

Academic Editor: Camile S. Farah

Copyright ( 2013 G. Prasad and M. McCullough. This is an open access article distributed under the Creative Commons Attribution License, which permits unrestricted use, distribution, and reproduction in any medium, provided the original work is properly cited.

\begin{abstract}
Chemokines have been shown to be important in both inflammation and carcinogenesis and are able to be measured in saliva with relatively robust methods including enzyme-linked immunosorbent assays (ELISA). Thus it has been hypothesized that patients with oral cancer and oral potentially malignant lesions will have elevated levels of specific chemokines in oral fluids and that this may be used as a marker of both the early detection of malignant disease and progression to malignancy. The concept that salivary biomarkers can be easily measured and indicate disease states has profound consequences for clinical practice and may open up new strategies for the diagnosis, prognosis, and potential therapy of oral squamous cell carcinoma (OSCC). This review focuses on our understanding of cytokines and chemokines and the potential role that they may have in clinical practice.
\end{abstract}

\section{Introduction}

Oral cancer is the eleventh most prevalent cancer worldwide [1]. Oral cancers in Australia account for approximately 2$3 \%$ of all cancers and approximately $1 \%$ of all cancer deaths, with an increasing incidence over the past decades [2]. The most common oral cancer is oral squamous cell carcinoma (OSCC), which makes up 90\% of all oral cancers [3], and if diagnosed early has a five-year survival rate of around $85 \%$ [4]. However, the early phase of oral cancer is often asymptomatic. Mortality for oral cancer is high because most patients seek care only when they experience late-stage symptoms (pain, persistent ulceration, unexplained bleeding, or an oral or neck mass), at which stage the disease is advanced and the survival rate decreases as low as $15-50 \%$. Early detection of oral cancer is therefore paramount for improving survival rates and prognosis for patients with the disease.

Current diagnostic techniques focus on detection of malignant and potentially premalignant lesions in the oral cavity. Early lesions may present as unhealing lesions, mucosal colour changes, pain, tenderness or numbness, protuberances, or rough, thickened, crusted, or eroded areas [5]. Typically, premalignant and malignant lesions begin as a subtle red or white patch (erythroplakia or leukoplakia) that eventually ulcerates and progresses to an exophytic mass [6]. Regular comprehensive examinations of the oral cavity form the backbone of oral cancer screening and are especially critical in patients with identified risk habits and factors such as tobacco smoking, excessive alcohol consumption, and human papilloma virus infection [7].

The advantage of the standard visual and tactile examination is that it is simple to perform and requires no added equipment. However, subtle lesions may pass undetected, and it is difficult to make a visual distinction between benign, premalignant, and malignant lesions. Adjunctive techniques have been developed in recent years to facilitate making this distinction and enhance the effectiveness of oral examinations. Techniques such as vital staining (Toluidine Blue) and visualisation adjuncts (VELscope and ViziLite) highlight abnormal mucosa by targeting tissues undergoing rapid cell division and areas of high metabolic turnover [8]. Another adjunctive technique employs transepithelial sampling of the oral mucosa for cytologic analysis (OralCDx Brush Test system). While promising, these emergent technologies have yet to reproduce the sensitivity and specificity of examination via tissue biopsy and histopathological examination, which remains the gold standard for oral cancer diagnosis [8]. 
A new focus of research is the use of salivary diagnostics for early detection of OSCC, which have the advantage of being noninvasive and nontoxic. Proteins, mRNA, enzymes, and chemicals extracted from saliva have been found at sufficiently distinct levels between OSCC and control samples to be considered as potential biomarkers [9]. These biomarkers could be important indicators of physiological or pathological states and provide information for the detection of early and differential markers for disease. Salivary biomarkers offer an easy, inexpensive, safe, and noninvasive approach for disease detection [10]. They have the potential to serve as a widely available screening tool that does not rely on the localization of a lesion for diagnosis [11]. This advantage over other detection methods gives salivary biomarker screening the potential to identify patients with malignant and potentially malignant lesions.

Recent studies have assessed variation in biomarkers in patients with oral cancer. Using an array of biomarkers from oral rinses from 40 HNSCC patients and 39 controls assessed by ELISA assays, it has been shown that it is possible to distinguish HNSCC cases from controls, particularly when the patients demographics were also considered [12]. Further, extensive analyses of the plasma levels of 48 proteins (26 cytokines, 10 chemokines, and 12 growth factors) in 111 untreated OSCC patients, 112 healthy individuals, and 107 individuals with potentially malignant oral mucosal lesions showed that the levels of 12 proteins were significantly dysregulated in OSCC patients serum [13]. Furthermore, a recent extensive study had been undertaken to substantiate the development of salivary biomarkers. This study assessed a panel of putative OSCC markers in 395 subjects in 5 independent validation cohorts and found them to be independently validated, reproducible, and robust for use in a reference laboratory [14]. Thus, such studies indicate the potential of specific deregulated proteins as predictive biomarkers in oral cancer. This review will discuss the potential of cytokines and chemokines as salivary biomarkers for the early diagnosis of oral squamous cell carcinoma.

\section{Cytokines}

Cytokines are a group of small, mainly secreted proteins that affect the behaviour of cells in a diverse number of ways. The binding of cytokines to specific cell membrane cytokine receptors can induce a number of activities within the cell, such as growth, differentiation, or death [15]. Most cytokines have pleiotropic effects; however, some are generally considered as proinflammatory, such as interferon-gamma (IFN$\gamma$ ), tumour necrosis factor-alpha (TNF- $\alpha$ ), and interleukinlbeta (IL-1 $\beta$ ) [16-19], whereas others are associated with antiinflammatory effects, such as transforming growth factorbeta-1 (TGF- $\beta 1$ ) [20].

Over the last 5 years, a considerable effort has been undertaken to analyse the salivary proteome. A large number of nonredundant proteins have been recognised in saliva, with one study [21] reporting over 1400 and another [22] almost 2,000 , reflecting the diversity of salivary biomarker profiles that may identify and potentially aid in the management of a range of diseases [23].
Of particular interest has been the use of salivary cytokine levels as markers of both cell proliferation and oral cancer [24]. The most studied cytokines include epidermal growth factor (EGF), interleukins-6 and -8, vascular endothelial growth factor (VEGF), interleukins-4 and -10, tumour necrosis factor (TNF) and endothelin [24, 25].

Several studies have assessed interleukin-6 (IL-6), a multifunctional cytokine that participates in the inflammatory and immune responses and has been shown to promote the growth of cancer cells as well as associated with an increased rate of metastasis and an altered immune status [26-32]. Interestingly, IL-6 would appear to have different effects on different cell populations, stimulatory for some cell types while inhibitory for others [30]. IL-6 can promote tumor cell proliferation in several tumor cell lines, including human cervical carcinomas mediated cachexia [33]. In contrast, another study indicated that expression of IL-6 and its receptor can be inhibitory for cell proliferation and is correlated with good prognoses for patients with breast cancer [34]. IL-6 also has a demonstrable direct effect on cancer cells via inactivation of the p53 tumour suppressor gene as seen in human multiple myeloma cell line KAS 6/1. IL-6R overexpression was associated with larger tumors and more advanced histologic grade [35]. Irrespective of the role of IL-6, there is increasing evidence to support higher levels of IL- 6 in the saliva of patients with oral cancer, as well as oral potentially malignant lesions, than in normal controls [36]. In a recent trial of 29 consecutive patients being treated for oral cancer, it was shown that patients had much higher salivary concentration of IL- 6 than controls and that this concentration increased during the treatment period returning to baseline levels at discharge [36].

Other studies however have assessed a panel of proinflammatory cytokines as markers of malignancy [27, 37]. A recent study assessing the levels of IL- $1 \alpha$, IL-6, IL-8, VEGF$\alpha$, and TNF- $\alpha$ in saliva, measured using quantitative ELISA, was undertaken in a group of 18 patients with tongue SCC [38]. These salivary biomarkers were demonstrated to be increased in patients with oral cancer; significantly increased in a subgroup of patients with endophytic tongue cancer and IL-8 levels; particularly shown to correlate with poor prognosis; and intriguingly also found to be higher in control individuals who both smoked and consumed alcohol daily [38].

However, it should be noted that elevated levels of IL-6 and IL- 8 have also been detected in other studies in the saliva of patients with periodontitis $[39,40]$. The main limitation of this study is the relatively small sample size $(n=10)$; nevertheless, although IL-8 was found to be higher in patients with periodontitis than in healthy controls, it is detected at significantly much greater levels in patients with OSCC [41]. Yet if this is true, it should be possible to differentiate between an inflammatory process and a neoplastic process by the amount of IL- 6 and IL-8. The study in 2008 by ArellanoGarcia et al. has multiple important innovative aspects, including the fact that it showed that multiplex bead based assays were as effective as ELISA assays for quantification of proteins in saliva and that IL- 8 and IL-1beta were expressed at significantly higher levels in OSCC patients [41]. Although 
there were only 20 cancer patients, with 20 age and gender matched controls, this study nevertheless clearly indicated the potential of constituents of saliva as biomarkers for oral cancer.

A further study assessing salivary levels of TNF- $\alpha$, IL- $1 \alpha$, IL-6, and IL-8 in a group of nine patients with OSCC with matched healthy controls [42] attempted to assess the relative influence of periodontal inflammation by using a modified gingival index to matched patients and control samples [42]. It found that IL-6 was statistically significantly higher in patients with OSCC. Interestingly though, several patients were edentulous, and thus neither they nor their matched controls would help discriminate the role of periodontal inflammation in relative salivary chemokine level, a fact compounded by the small sample size ( 9 patients with OSCC) [42].

Thus far then, the results of a number of studies would indicate that salivary cytokine levels are very likely to provide useful information of the presence of disease, epithelial behaviour, the local inflammatory response, and carcinogenesis. However, larger sample sized studies are required to investigate salivary cytokines and their role in the diagnosis of PML and OSCC while at the same time being able to deal with the obvious local confounding factor of inflammation and in particular periodontal disease. Further studies of the potential of a panel of salivary cytokines as a screening tool for oral cancer are apparently ongoing, the results of which are eagerly awaited as this is likely to have a profound impact on the early detection of oral cancer and thus morbidity and mortality [11]. The complexity of undertaking such a study, that would require a large number of patients who have oral cancer, patients with potentially malignant mucosal disease, and sufficient health controls as well as patients with non-neoplastic mucosal disease. This comprehensive study, thoroughly analysing the diversity of these salivary biomarkers present in health and disease, is at the same time both daunting and necessary.

\section{Chemokines}

Chemokines are a superfamily of structurally related cytokines, which share an ability to chemotactically attract their target cells along a concentration gradient [43]. It is through this ability that these molecules play an integral role in the migration of immune cells to areas of pathogen challenge. Chemokines also mediate the movement of specific cells involved in inflammatory responses that subsequently result in cellular interactions critical for mounting immune responses [43].

All chemokines are small proteins, ranging in weight from 6 to $14 \mathrm{KDa}$. There are now over 50 identified chemokines and 20 chemokine receptors [44]. Chemokines and chemokine receptors can be classified into 4 main structural families, dependent upon the position of the cysteine residues near the $\mathrm{N}$-terminus. These families are the $\mathrm{CC}$, $\mathrm{CXC}, \mathrm{C}$, and $\mathrm{CX} 3 \mathrm{C}$, with the $\mathrm{X}$ denoting the number of amino acids between the cysteine residues $[45,46]$.

Chemokines are secreted in response to signals such as proinflammatory cytokines such as interleukin (IL)-1, tumor necrosis factor (TNF), and interferon-c (IFN-c) and thus they play an important role in selectively recruiting monocytes, neutrophils, and lymphocytes [47].

Once induced, the directed migration of cells expressing the appropriate chemokine receptors occurs along a chemical ligand gradient known as the chemokine gradient. This allows cells to move toward high local concentrations of chemokines [48]. Chemokines induce chemotaxis through the activation of G-protein-coupled receptors (GPCRs), subsequently involving adhesion molecules and glycosaminoglycans (GAGs) [49]. Chemokines bind to specific cell surface transmembrane receptors coupled with heterotrimeric G proteins, whose activation leads to the activation of intracellular signaling cascades that prompt migration toward the chemokine source (chemotaxis) [50]. This interaction results in multiple signal transduction pathways being activated. One of the characteristics associated with the chemokine system is its redundancy. It has been shown that a single ligand can bind to multiple receptors and in turn a chemokine receptor may bind multiple ligands [51]. Also, many of the inflammatory chemokines have wide target cell selectivity, with some acting both on the cells of the innate and adaptive immunity [51].

The function of chemokines can be subdivided into two main families: those that are induced after inflammatory stimuli, the inflammatory chemokines, and those produced constitutively in tissues, the homing chemokines [52]. In addition to their roles in the immune system, chemokines and chemokine receptors are also involved in the pathology of a number of diseases, such as infections (e.g., HIV-1/AIDS), autoimmune disorders (e.g., psoriasis, rheumatoid arthritis, and multiple sclerosis), pulmonary diseases (asthma and chronic obstructive pulmonary disease), transplant rejection, cancer, and vascular disease [53]. Furthermore, there would appear to be significant overlap between chemokines as some of the inflammatory chemokines appear to be produced constitutively in some areas of the body [54] and some of the chemokines designated as homing chemokines can be upregulated by inflammatory stimuli [55]. For example, LARC/MIP- $3 \alpha$ plays a role in both homeostatic trafficking of leukocytes, as well acting as an inflammatory chemokine during host defense [56].

Although the detection of chemokine levels by enzymelinked immunosorbent assay (ELISA) has become a sensitive and specific method to determine the chemokine profile in patient fluids, this is not able to fully represent the actual inflammatory conditions in vivo. Indeed, many chemokines are posttranslationally modified by proteolytic cleavage, which can render an agonist more active or inactive or even convert the active chemokine into a receptor antagonist of the intact molecule [57].

Nevertheless, using ELISA, a recent study assessed the saliva of patients with oral cancer for the presence of both inflammatory chemokines (CXCL8, CXCL10, and CCL2), homeostatic chemokines (CXCL4, CCL14, and CCL18) [58]. Further, individuals with and without periodontitis were used as controls and it was found that $\mathrm{H} \& \mathrm{~N}$ carcinomas give rise to a change in the chemokine composition of the oral fluid with a significant increase in CXCL8, CXCL10, and 
CCL14 before therapy, a finding that was not reproduced after therapy [58]. However, the levels detectable by ELISA were very low and it is likely that more refined methods could indicate not only intact chemokines, but also those modified posttranslationally [58]. These authors conclude that it can be expected that specific truncated chemokines, as well as the proteases involved in this truncation, will be linked to particular disease states. These authors postulate that proteomic analysis of biological fluids will further our understanding of the pathogenesis of specific diseases and provide solutions for new diagnostic and treatment options [58]. Since chemokines in disease can be occasionally involved in excessive recruitment of inflammatory cells, prevention of this recruitment may be an effective antiinflammatory strategy. Furthermore, chemokine receptors are intimately involved in cellular recruitment and, along with CD4, have been shown to be an essential cofactor enabling HIV-1 viruses to infect cells [59]. Thus, in diseases that have a profound effect on the immune system, such as infection with HIV, there are various points of potential intervention that could provide anti-inflammatory and antiHIV infectivity therapeutics, including prevention of the receptor-ligand interaction, prevention of the chemokineglycosaminoglycan interaction, interfering with the signaling pathways that are induced upon receptor activation, and modification of receptor trafficking pathways [59].

However, these postulated potential interventions need considerable further study as the apparent redundancy in the expression of chemokines, and the overlap between homeostatic and inflammatory chemokines pathways, makes them difficult targets for diagnosis of diseases and therapeutics. In addition, it has been shown that the enhancement of the inflammatory response is aided by synergistic activity of chemokines for leukocyte migration [60]. Hence, blockage of a single chemokine may downregulate other immune responses, because of the inhibitory effect on its synergy with other chemokines [60]. Thus, there is a need to refine our ability to assess the presence of chemokines in disease states, the presence and specificity of chemokine receptors, and the specificity of functional active chemokines in specific disease states, prior to being able to define selective and specific targets for treatment.

Significant change in our understanding of the role of chemokines in OSCC has occurred in a fairly short time. A relatively early study assessed the presence of a particular chemokine (CXCL12) and its specific receptor (CXCR4), revealing that the receptor was more prevalent in oral cancers that metastasized, suggesting that this chemokine/receptor may be important in the regulation of tumour growth and organ-specific lymphatic spread [61]. A further, extensive study of 85 patients with oral SCCC utilized immunohistochemistry, RT-PCR, and western blot to assess the expression of a different chemokine, CCR7 and its ligand CCL21, in 85 patients with oral squamous cell carcinoma [10]. It was shown that CCR7 expression was positively correlated with lymph node metastasis, tumour size, and clinical stage, and these authors postulated that the interaction between this chemokine and its receptor may be significant for the induction of lymphatic spread.
The mechanism by which chemokines and chemokine receptors are involved in oral carcinogenesis has been extensively studied [19, 62-64]. CCL5 (previously known as RANTES-Regulated on Activation, Normal T cell Expressed and Secreted) has been shown to play a crucial role in migration and metastasis in human cancer cell lines and further showed that that CCL5/CCR5 axis enhanced migration of oral cancer cells, probably via MMP-9 [65]. An extensive investigation of 253 oral cancer patients, matched with 347 controls, the presence of mutations (single nucleotide polymorphisms) in the genes of specific chemokine ligands and receptors (CCL5 and CCR5) revealed an interesting dichotomy of the presence of mutations increasing risk for oral cancer while at the same time raising the potential that oral cancers with a specific chemokine profile may well have enhanced protection from metastases [66]. In a bid to rectify the dysregulated CC chemokine receptor (CCR5)/ligand, a recent study used interferon- $\alpha 2 \mathrm{~b}$ (IFN- $\alpha 2 \mathrm{~b}$ ), known to upregulate CCR5 expression [67], in a small cohort of 12 oral cancer patients. These investigators showed that enhanced T-cell-mediated tumor cell killing upon IFN- $\alpha 2 \mathrm{~b}$ treatment and they postulate that this immunotherapy treatment may be combined with standard chemotherapy for better clinical outcome [67].

The SDF-1/CXCR4 (stromal cell derived factor 1/chemokine (C-X-C motif) receptor 4 ) pathway has been suggested to play a role in the metastatic dissemination of neoplasms with migration toward SDF-1 by tumor cells bearing CXCR4. Mutation in the gene of a specific chemokine receptor (CXCR4) has been noted to have an increased likelihood of more advanced oral cancer (stage III and IV by 2.66 -fold) [68]. A study of 71 patients with HNSCC assessing the tissue expression levels of SDF-1 and CXCR4 found that patients with low SDF-1 had poorer metastasis-free survival $(P=$ 0.026), disease-free survival $(P=0.006)$ and overall survival rates $(P=0.002)[69]$. A recent immunohistochemical study has confirmed that this relationship showing a significant relationship between CXCL12 and CXCR4 was found both in potentially malignant lesions and oral cancer [70]. An in vitro experiment has recently shown that, with synthetic biology approaches, signalling selective inhibition of the CXCR4 prevented the metastatic spread of neoplastic cells [71].

Previously, investigations based on the known association between the chemokine ligand CXCL13 and prognosis of oral cancer found that the chemokine ligand/receptor axis of CXCL13/CXCR5 is not only important for cancer bone invasion and metastasis but may also be a potential therapeutic target to prevent OSCC bone invasion/osteolysis [72].

\section{Conclusion}

Current research has identified deregulated cytokines in OSCC as well as oral potentially malignant lesions, robust and reproducible methods for the assessment of these cytokines in saliva, and the possibility of a rapid salivary test as an indicator of disease and risk of malignancy. Ongoing development of such a method will have profound impact on oral cancer screening and the early diagnosis of oral cancer, 
potentially resulting in early treatment and a decrease in the high levels of morbidity and mortality associated with OSCC. Furthermore, there are indications that it may be possible to utilize our enhanced understanding of the chemokines associated with disease progression, metastases, and bone invasion to develop novel methods for the treatment of oral cancer.

\section{References}

[1] World Health Organisation (WHO), Global Data on Incidence of Oral Cancer, WHO/NMH/CHP/HPR/ORH, 2005.

[2] Australian Institute of Health Welfare (AIHW) and Australasian Association of Cancer Registries (AACR), Cancer in Australia: An Overview, Cancer series no. 46. Cat. no. CAN 42, AIHW, Canberra, Australia, 2008.

[3] B. W. Neville and T. A. Day, "Oral cancer and precancerous lesions," Cancer Journal for Clinicians, vol. 52, no. 4, pp. 195-215, 2002.

[4] J. J. Sciubba, "Oral cancer and its detection: history-taking and the diagnostic phase of management," Journal of the American Dental Association, vol. 132, no. 11, pp. 12S-18S, 2001.

[5] J. F. Carew, B. Singh, and J. P. Shah, "Clinical evaluation and differential diagnosis," Oral Cancer, pp. 183-198, 2003.

[6] W. C. Gonsalves, A. C. Chi, and B. W. Neville, "Common oral lesions: part II. Masses and neoplasia," The American Family Physician, vol. 75, no. 4, pp. 509-512, 2007.

[7] M. J. McCullough, G. Prasad, and C. S. Farah, "Oral mucosal malignancy and potentially malignant lesions: an update on the epidemiology, risk factors, diagnosis and management," Australian Dental Journal, vol. 55, pp. 61-65, 2010.

[8] L. L. Patton, J. B. Epstein, and A. R. Kerr, "Adjunctive techniques for oral cancer examination and lesion diagnosis a systematic review of the literature," The Journal of the American Dental Association, vol. 139, no. 7, pp. 896-905, 2008.

[9] Y. S. L. Cheng and J. Wright, "Advances in diagnostic adjuncts for oral squamous cell carcinoma," The Open Pathology Journal, vol. 5, pp. 3-7, 2011.

[10] A. Zhang, H. Sun, P. Wang, and X. Wang, "Salivary proteomics in biomedical research," Clinica Chimica Acta, vol. 415, pp. 261265, 2013.

[11] D. T. W. Wong, "Salivaomics," The Journal of the American Dental Association, vol. 143, pp. 19S-24S, 2012.

[12] L. H. Pereira, I. N. Adebisi, A. Perez et al., "Salivary markers and risk factor data: a multivariate modeling approach for head and neck squamous cell carcinoma detection," Cancer Biomarkers, vol. 10, no. 5, pp. 241-249, 2011.

[13] K.-P. Chang, Y.-T. Chang, C.-T. Liao et al., "Prognostic cytokine markers in peripheral blood for oral cavity squamous cell carcinoma identified by multiplexed immunobead-based profiling," Clinica Chimica Acta, vol. 412, no. 11-12, pp. 980-987, 2011.

[14] D. Elashoff, H. Zhou, J. Reiss et al., "Prevalidation of salivary biomarkers for oral cancer detection," Cancer Epidemiology Biomarkers and Prevention, vol. 21, no. 4, pp. 664-672, 2012.

[15] C. A. Janeway Jr., P. Travers, M. Walport, and M. J. Shlomchik, Immunobiology: The Immune System in Health and Disease, Churchill Livingstone, 1996.

[16] C. A. Dinarello, "Role of pro- and anti-inflammatory cytokines during inflammation: experimental and clinical findings," Journal of Biological Regulators and Homeostatic Agents, vol. 11, no. 3, pp. 91-103, 1997.
[17] M. R. Piva, L. B. de Souza, P. R. Martins-Filho et al., "Role of inflammation in oral carcinogenesis (part II): CD8, FOXP3, TNF- $\alpha$, TGF- $\beta$ and NF- $\kappa$ B expression," Oncology Letters, vol. 5, no. 6, pp. 1909-1914, 2013.

[18] N. L. Costa, M. C. Valadares, P. P. Souza et al., "Tumor-associated macrophages and the profile of inflammatory cytokines in oral squamous cell carcinoma," Oncology Letters, vol. 49, no. 3, pp. 216-223, 2013.

[19] S. Koontongkaew, “The tumor microenvironment contribution to development, growth, invasion and metastasis of head and neck squamous cell carcinomas," Journal of Cancer, vol. 4, no. 1, pp. 66-83, 2013.

[20] E. Ling and D. S. Robinson, “Transforming growth factor- $\beta 1$ : its anti-inflammatory and pro-fibrotic effects," Clinical and Experimental Allergy, vol. 32, no. 2, pp. 175-178, 2002.

[21] E. Scarano, A. Fiorita, P. M. Picciotti et al., "Proteomics of saliva: personal experience," Acta Otorhinolaryngologica Italica, vol. 30, no. 3, pp. 125-130, 2010.

[22] H. Xiao and D. T. Wong, "Proteomics and its applications for biomarker discovery in human saliva," Bioinformation, vol. 5, no. 7, pp. 294-296, 2011.

[23] S. Bandhakavi, M. D. Stone, G. Onsongo, S. K. van Riper, and T. J. Griffin, "A dynamic range compression and three-dimensional peptide fractionation analysis platform expands proteome coverage and the diagnostic potential of whole saliva," Journal of Proteome Research, vol. 8, no. 12, pp. 5590-5600, 2009.

[24] M. Schapher, O. Wendler, and M. Gröschl, "Salivary cytokines in cell proliferation and cancer," Clinica Chimica Acta, vol. 412, no. 19-20, pp. 1740-1748, 2011.

[25] Z. Culig, "Interleukin-6 as a therapy target in oral squamous carcinoma," Expert Opinion on Therapeutic Targets, vol. 17, no. 1, pp. 53-59, 2013.

[26] M. A. St John, Y. Li, X. Zhou et al., "Interleukin 6 and interleukin 8 as potential biomarkers for oral cavity and oropharyngeal squamous cell carcinoma," Archives of Otolaryngology, vol. 130, no. 8, pp. 929-935, 2004.

[27] Z. Chen, P. S. Malhotra, G. R. Thomas et al., "Expression of proinflammatory and proangiogenic cytokines in patients with head and neck cancer," Clinical Cancer Research, vol. 5, no. 6, pp. 1369-1379, 1999.

[28] E. A. Mann, J. D. Spiro, L. L. Chen, and D. L. Kreutzer, "Cytokine expression by head and neck squamous cell carcinomas," The American Journal of Surgery, vol. 164, no. 6, pp. 567-573, 1992.

[29] K. V. Woods, A. El-Naggar, G. L. Clayman, and E. A. Grimm, "Variable expression of cytokines in human head and neck squamous cell carcinoma cell lines and consistent expression in surgical specimens," Cancer Research, vol. 58, no. 14, pp. 31323141, 1998.

[30] Y.-F. Wang, S.-Y. Chang, S.-K. Tai, W.-Y. Li, and L.-S. Wang, "Clinical significance of interleukin-6 and interleukin-6 receptor expressions in oral squamous cell carcinoma," Head and Neck, vol. 24, no. 9, pp. 850-858, 2002.

[31] L. Nibali, S. Fedele, F. D’Aiuto, and N. Donos, "Interleukin-6 in oral diseases: a review," Oral Diseases, vol. 18, no. 3, pp. 236-243, 2012.

[32] Y. S. Hwang, S. K. Lee, K.-K. Park, and W.-Y. Chung, "Secretion of IL-6 and IL-8 from lysophosphatidic acid-stimulated oral squamous cell carcinoma promotes osteoclastogenesis and bone resorption," Oral Oncology, vol. 48, no. 1, pp. 40-48, 2012.

[33] S. Tamura, K. F. Ouchi, K. Mori et al., "Involvement of human interleukin 6 in experimental cachexia induced by a human 
uterine cervical carcinoma xenograft," Clinical Cancer Research, vol. 1, no. 11, pp. 1353-1358, 1995.

[34] A. Karczewska, S. Nawrocki, D. Breborowicz, V. Filas, and A. Mackiewicz, "Expression of interleukin-6, interleukin-6 receptor, and glycoprotein 130 correlates with good prognoses for patients with breast carcinoma," Cancer, vol. 88, no. 9, pp. 2061-2071, 2000.

[35] D. R. Hodge, B. Peng, J. C. Cherry et al., "Interleukin 6 supports the maintenance of p53 tumor suppressor gene promoter methylation," Cancer Research, vol. 65, no. 11, pp. 4673-4682, 2005.

[36] J. Sato, J. Goto, T. Murata et al., "Changes in saliva interleukin-6 levels in patients with oral squamous cell carcinoma," Oral Surgery, Oral Medicine, Oral Pathology, Oral Radiology and Endodontology, vol. 110, no. 3, pp. 330-336, 2010.

[37] M. J. McNamara, H. R. Alexander, and J. A. Norton, "Cytokines and their role in the pathophysiology of cancer cachexia," Journal of Parenteral and Enteral Nutrition, vol. 16, no. 6, pp. 50S55S, 1992.

[38] A. Korostoff, L. Reder, R. Masood, and U. K. Sinha, "The role of salivary cytokine biomarkers in tongue cancer invasion and mortality," Oral Oncology, vol. 47, no. 4, pp. 282-287, 2011.

[39] C. Giannopoulou, J. J. Kamma, and A. Mombelli, "Effect of inflammation, smoking and stress on gingival crevicular fluid cytokine level," Journal of Clinical Periodontology, vol. 30, no. 2, pp. 145-153, 2003.

[40] P.-L. Wang, K. Ohura, T. Fujii et al., "DNA microarray analysis of human gingival fibroblasts from healthy and inflammatory gingival tissues," Biochemical and Biophysical Research Communications, vol. 305, no. 4, pp. 970-973, 2003.

[41] M. E. Arellano-Garcia, S. Hu, J. Wang et al., "Multiplexed immunobead-based assay for detection of oral cancer protein biomarkers in saliva," Oral Diseases, vol. 14, no. 8, pp. 705-712, 2008.

[42] M. Sahebjamee, M. Eslami, F. Atarbashimoghadam, and A. Sarafnejad, "Salivary concentration of TNF $\alpha$, IL1 $\alpha$, IL6, and IL8 in oral squamous cell carcinoma," Medicina Oral Patologia Oral y Cirugia Bucal, vol. 13, no. 5, pp. 292-295, 2008.

[43] A. Zlotnik and O. Yoshie, "Chemokines: a new classification system and their role in immunity," Immunity, vol. 12, no. 2, pp. 121$127,2000$.

[44] P. A. Ruffini, P. Morandi, N. Cabioglu, K. Altundag, and M. Cristofanilli, "Manipulating the chemokine-chemokine receptor network to treat cancer," Cancer, vol. 109, no. 12, pp. 2392-2404, 2007.

[45] T. S. Olson and K. Ley, "Chemokines and chemokine receptors in leukocyte trafficking," The American Journal of PhysiologyRegulatory, Integrative and Comparative Physiology, vol. 283, no. 1, pp. R7-R28, 2002.

[46] V. Vinader and K. Afarinkia, "Mini focus: chemokines-review a beginner's guide to chemokines," Future Medicinal Chemistry, vol. 4, no. 7, pp. 845-852, 2012.

[47] M. Gouwy, S. Struyf, P. Proost, and J. van Damme, "Synergy in cytokine and chemokine networks amplifies the inflammatory response," Cytokine and Growth Factor Reviews, vol. 16, no. 6, pp. 561-580, 2005.

[48] C. Callewaere, G. Banisadr, W. Rostène, and S. M. Parsadaniantz, "Chemokines and chemokine receptors in the brain: implication in neuroendocrine regulation," Journal of Molecular Endocrinology, vol. 38, no. 3-4, pp. 355-363, 2007.
[49] S. J. Hyduk, J. R. Chan, S. T. Duffy et al., "Phospholipase C, calcium, and calmodulin are critical for $\alpha 4 \beta 1$ integrin affinity upregulation and monocyte arrest triggered by chemoattractants," Blood, vol. 109, no. 1, pp. 176-184, 2007.

[50] F. Sallusto and M. Baggiolini, "Chemokines and leukocyte traffic," Nature Immunology, vol. 9, no. 9, pp. 949-952, 2008.

[51] R. Guerreiro, Q. Santos-Costa, and J. M. Azevedo-Pereira, "The chemokines and their receptors: characteristics and physiological functions," Acta Médica Portuguesa, vol. 24, no. 4, pp. 967976, 2011.

[52] E. J. Kunkel and E. C. Butcher, "Chemokines and the tissuespecific migration of lymphocytes," Immunity, vol. 16, no. 1, pp. $1-4,2002$.

[53] S. L. Deshmane, S. Kremlev, S. Amini, and B. E. Sawaya, "Monocyte chemoattractant protein-1 (MCP-1): an overview," Journal of Interferon and Cytokine Research, vol. 29, no. 6, pp. 313-326, 2009.

[54] A. Izadpanah, M. B. Dwinell, L. Eckmann, N. M. Varki, and M. F. Kagnoff, "Regulated MIP-3alpha/CCL20 production by human intestinal epithelium: mechanism for modulating mucosal immunity", The American Journal of PhysiologyGastrointestinal and Liver Physiology, vol. 280, no. 4, pp. G710G719, 2001.

[55] J. Morales, B. Homey, A. P. Vicari et al., "CTACK, a skin-associated chemokine that preferentially attracts skin-homing memory T cells," Proceedings of the National Academy of Sciences of the United States of America, vol. 96, no. 25, pp. 14470-14475, 1999.

[56] E. Schutyser, S. Struyf, P. Menten et al., "Regulated production and molecular diversity of human liver and activation-regulated chemokine/macrophage inflammatory protein- $3 \alpha$ from normal and transformed cells," Journal of Immunology, vol. 165, no. 8, pp. 4470-4477, 2000.

[57] S. Struyf, P. Proost, and J. van Damme, "Regulation of the immune response by the interaction of chemokines and proteases," Advances in Immunology, vol. 81, pp. 1-44, 2003.

[58] K. Michiels, E. Schutyser, R. Conings et al., "Carcinoma cellderived chemokines and their presence in oral fluid," European Journal of Oral Sciences, vol. 117, no. 4, pp. 362-368, 2009.

[59] A. E. I. Proudfoot, C. A. Power, C. Rommel, and T. N. C. Wells, "Strategies for chemokine antagonists as therapeutics," Seminars in Immunology, vol. 15, no. 1, pp. 57-65, 2003.

[60] M. Gouwy, S. Struyf, S. Noppen et al., "Synergy between coproduced CC and CXC chemokines in monocyte chemotaxis through receptor-mediated events," Molecular Pharmacology, vol. 74, no. 2, pp. 485-495, 2008.

[61] H. H. Oliveira-Neto, P. P. de Souza, M. R. da Silva, E. F. Mendonça, T. A. Silva, and A. C. Batista, "The expression of chemokines CCL19, CCL21 and their receptor CCR7 in oral squamous cell carcinoma and its relevance to cervical lymph node metastasis," Tumor Biology, vol. 34, no. 1, pp. 65-70, 2013.

[62] E. Oue, J. W. Lee, K. Sakamoto et al., "CXCL2 synthesized by oral squamous cell carcinoma is involved in cancer-associated bone destruction," Biochemical and Biophysical Research Communications, vol. 424, no. 3, pp. 456-461, 2012.

[63] W. A. Yeudall, C. A. Vaughan, H. Miyazaki et al., "Gain-offunction mutant p53 upregulates CXC chemokines and enhances cell migration," Carcinogenesis, vol. 33, no. 2, pp. 442-451, 2012.

[64] C. Thode, T. G. Jørgensen, E. Dabelsteen, I. Mackenzie, and S. Dabelsteen, "Significance of myofibroblasts in oral squamous 
cell carcinoma," Journal of Oral Pathology and Medicine, vol. 40, no. 3, pp. 201-207, 2011.

[65] J.-Y. Chuang, W.-H. Yang, H.-T. Chen et al., "CCL5/CCR5 axis promotes the motility of human oral cancer cells," Journal of Cellular Physiology, vol. 220, no. 2, pp. 418-426, 2009.

[66] C. J. Weng, M. H. Chien, C. W. Lin et al., "Effect of CC chemokine ligand 5 and $\mathrm{CC}$ chemokine receptor 5 genes polymorphisms on the risk and clinicopathological development of oral cancer," Oral Oncology, vol. 46, no. 10, pp. 767-772, 2010.

[67] K. Chakraborty, A. Bose, K. K. Goswami et al., "Dysregulated $\mathrm{CC}$ receptor/ligand in monocytes/macrophages from tongue squamous cell carcinoma patients is partially rectified by interferon $\alpha$-2b," Human Immunology, vol. 73, no. 1, pp. 38-47, 2012.

[68] Y.-H. Teng, T.-H. Liu, H.-C. Tseng et al., "Contribution of genetic polymorphisms of stromal cell-derived factor-1 and its receptor, CXCR4, to the susceptibility and clinicopathologic development of oral cancer," Head and Neck, vol. 31, no. 10, pp. 12821288, 2009.

[69] F. Clatot, J.-M. Picquenot, O. Choussy et al., "Intratumoural level of SDF-1 correlates with survival in head and neck squamous cell carcinoma," Oral Oncology, vol. 47, no. 11, pp. 10621068, 2011.

[70] J. Xia, N. Chen, Y. Hong et al., "Expressions of CXCL12/CXCR4 in oral premalignant and malignant lesions," Mediators of Inflammation, vol. 2012, Article ID 516395, 5 pages, 2012.

[71] H. Yagi, W. Tan, P. Dillenburg-Pilla et al., "Cancer biology: a synthetic biology approach reveals a CXCR4-G13-rho signaling axis driving transendothelial migration of metastatic breast cancer cells," Science Signaling, vol. 4, no. 191, article ra60, 2011.

[72] S. Yuvaraj, A. C. Griffin, K. Sundaram, K. L. Kirkwood, J. S. Norris, and S. V. Reddy, "A novel function of CXCL13 to stimulate RANK ligand expression in oral squamous cell carcinoma cells," Molecular Cancer Research, vol. 7, no. 8, pp. 1399-1407, 2009. 


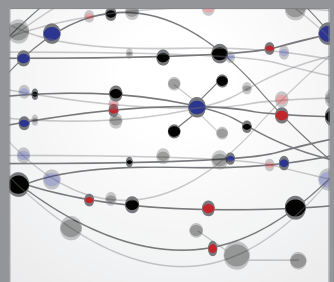

The Scientific World Journal
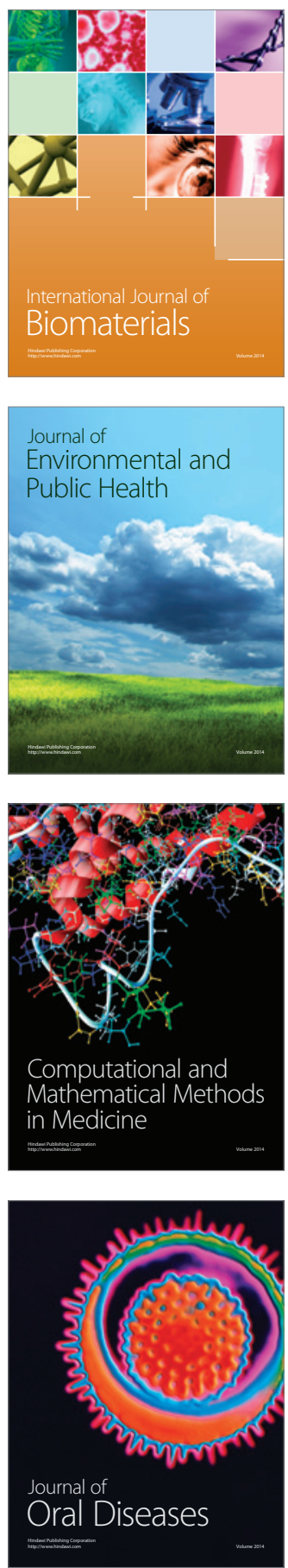
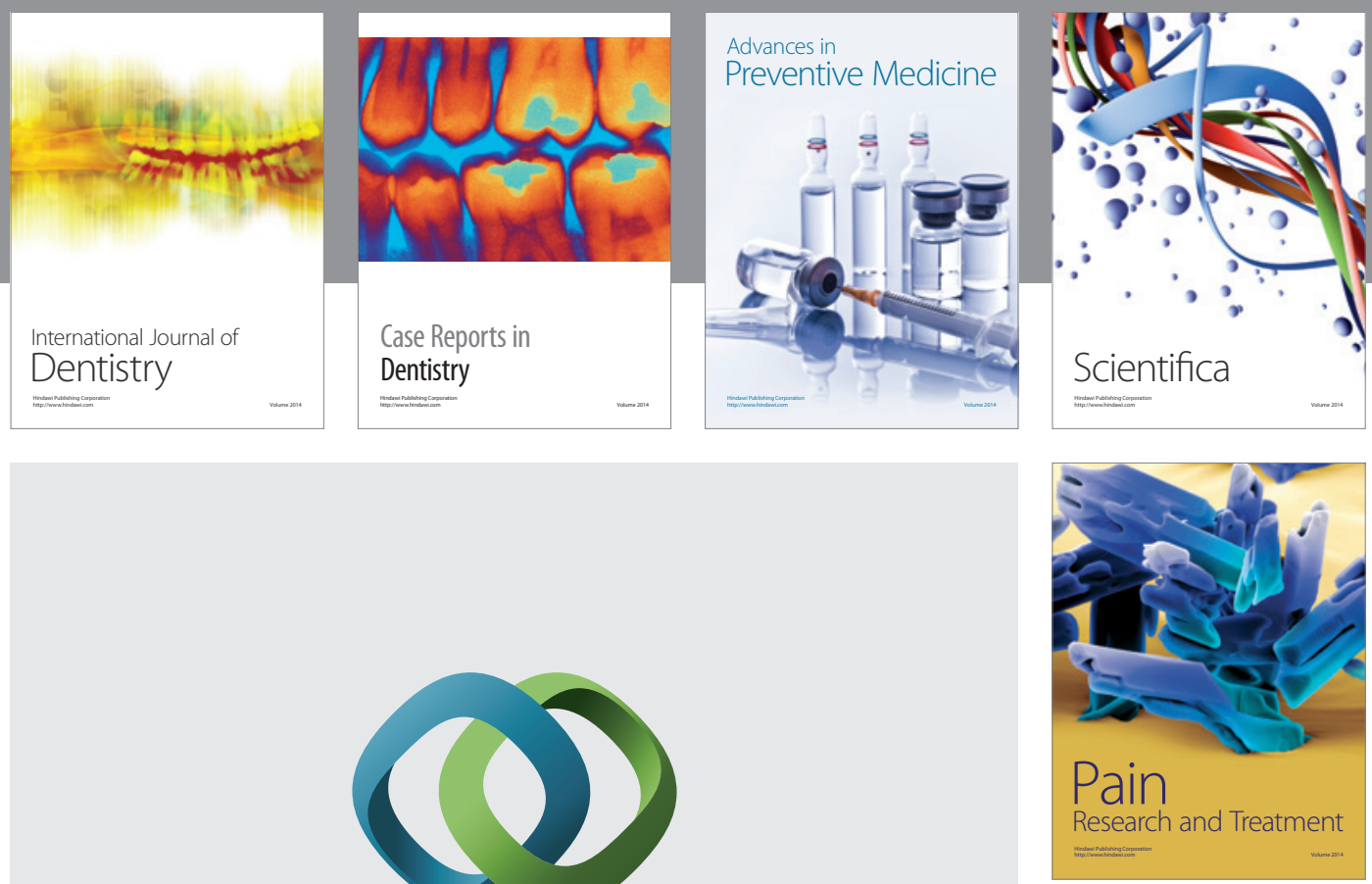

\section{Hindawi}

Submit your manuscripts at

http://www.hindawi.com
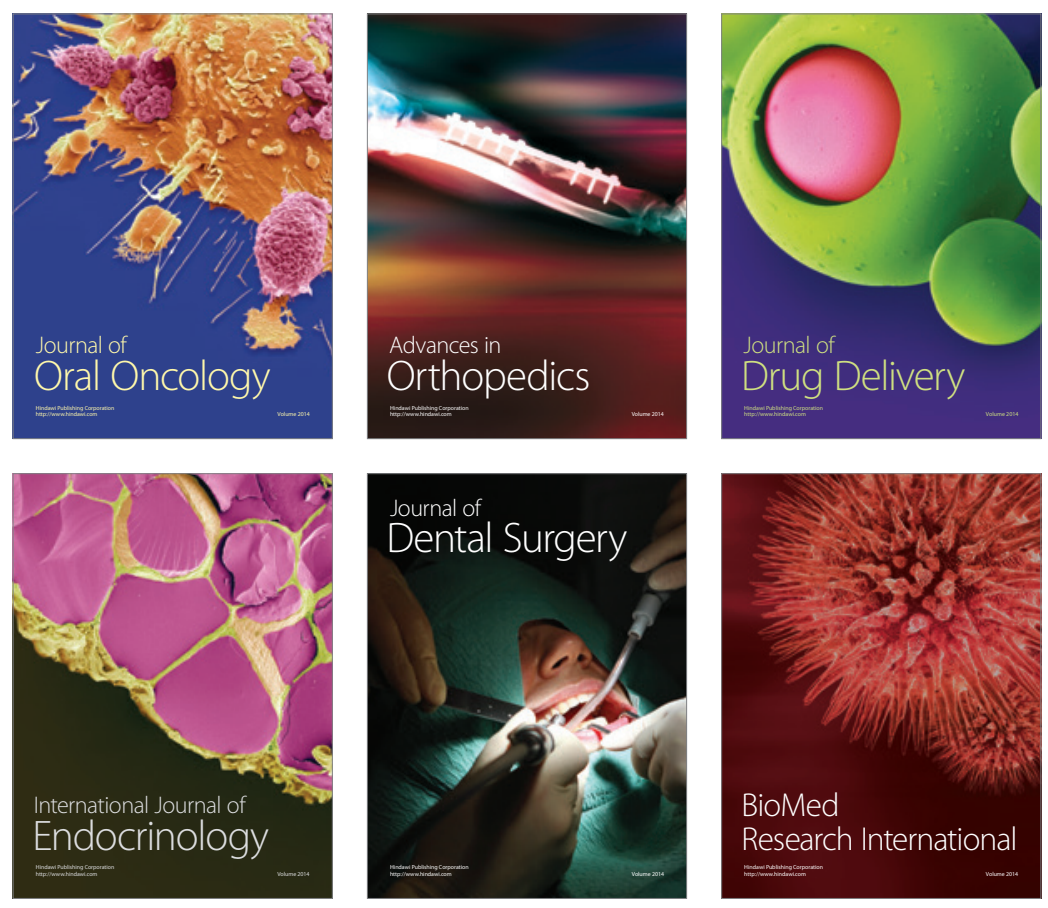

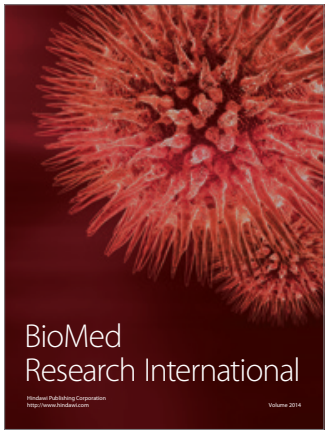

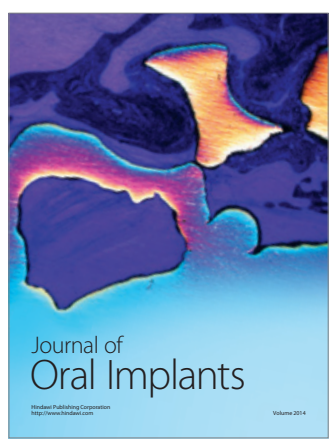
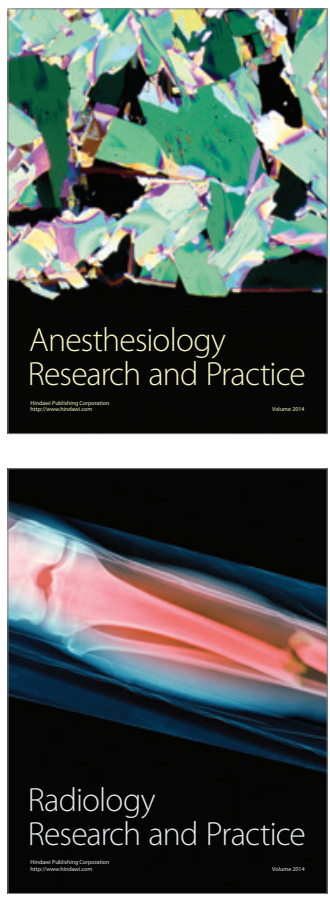